\title{
APLICACIÓN DEL MÉTODO DE RESISTIVIDAD ELÉCTRICA EN LA EXPLORACIÓN DE YACIMIENTOS DE CARBÓN
}

\author{
Application de la méthode de résistivité électrique dans \\ l'exploration des gisements de charbon
}

\section{EPISTEMUS}

ISSN: 2007-8196 (electrónico)

ISSN: 2007-4530 (impresa)

Francisco Miguel Oliver Ocaño ${ }^{1}$

Jesús Alberto Laborín Moreno ${ }^{2}$

Francisco Javier Becuar Peña ${ }^{3}$

Carlos Francisco García López 4

Recibido: 12 de septiembre de 2019,

Aceptado: 30 de noviembre del 2019

Autor de Correspondencia:

MTC Francisco Miguel Oliver Ocaño

Correo:miguel.oliver@unison.mx

\section{Resumen}

El método de resistividad de corriente directa es una técnica utilizada en la prospección minera, geohidrológica, geotérmica y en problemas de geotecnia. Este método se basa en la inyección de corriente eléctrica (directa o de baja frecuencia) en el terreno y en la medición de las diferencias de potencial asociadas al paso de la corriente a través de zonas del subsuelo con distinta conductividad eléctrica. En este trabajo se implementó este método utilizando arreglos multielectrodo tipo Dipolo-Dipolo y Schlumberger, en un lote Minero en San Javier, Sonora; con el objetivo de determinar la posible presencia de anomalías asociadas a carbón mineral en el subsuelo.

Palabras clave: Resistividad, Dipolo-Dipolo, Schlumberger, carbón.

\section{Abstract}

The direct current resistivity method is a technique used in mining, geohydrological, geothermal and geotechnical prospecting. This method is based on the injection of electric current (direct or low frequency) in the ground and on the measurement of the potential differences associated with the passage of the current through subsoil areas with different electrical conductivity. In this work, this method was implemented using Dipolo-Dipolo and Schlumberger multielectrode arrangements, in a mining lot in San Javier, Sonora; with the objective of determining the possible presence of anomalies associated with coal in the subsoil.

Keywords: Resistivity, Dipolo-Dipolo, Schlumberger, coal.

1 MTC Universidad de Sonora/miguel.oliver@unison.mx 


\section{INTRODUCCIÓN}

El arreglo electródico Dipolo-Dipolo se utiliza ampliamente para encontrar variaciones laterales de resistividad eléctrica asociadas con la presencia de cuerpos mineralizados entre otras aplicaciones. Este método, consiste en la medición a lo largo de un perfil, de diferencias de potencial en la superficie causadas por corrientes galvánicas que se inyectan al subsuelo a través de un par de electrodos (varillas de acero) (Newman et al., 2008).

El equipo utilizado para la obtención de estos datos, consistió de un resistivímetro con una corriente de salida Máxima de 10 Amperes y 1100 volts distribuidos en 11 pasos de 100 volts, conectado a un motogenerador de 3500 Watts de potencia; como receptor se utilizó un multímetro digital; así como todos los accesorios respectivos al equipo de campo (cables, carretes, electrodos no polarizables, varillas de acero, etc.). Los electrodos de medición se ubicaron utilizando un GPS diferencial marca Trimble doble frecuencia con sistema de medición RTK.

La geología dentro del área de estudio es muy compleja, las rocas que afloran en los alrededores corresponden a rocas sedimentarias constituidas de areniscas y lutitas, en cuyos estratos se localizan las vetas de Carbón Mineral. Estas rocas se distribuyen a todo lo largo del área de estudio, en donde también afloran rocas ígneas compuestas por andesitas y tobas andesíticas, las cuales sobreyacen a las rocas sedimentarias. El área se encuentra estructuralmente muy plegada y con diversas Fallas, lo que ha interrumpido la secuencia de las vetas de carbón dentro de las minas en explotación (INEGI).

Por estas razones, se realizó un estudio geofísico que consistió de un levantamiento de un perfil de resistividad eléctrica (Tomografía eléctrica) utilizando el arreglo multielectrodo tipo Dipolo-Dipolo, en terrenos de un lote Minero ubicado en el municipio de San Javier, Sonora (Figura 1); con el objetivo de determinar la posible presencia en el subsuelo de anomalías asociadas a carbón mineral hasta una profundidad de investigación estimada

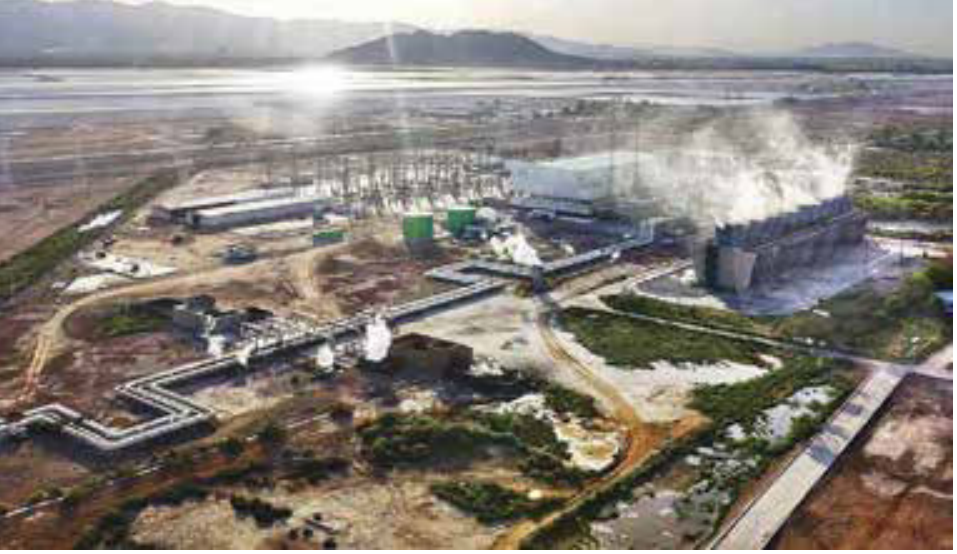

de 100 metros. También, para conocer la continuación del yacimiento en explotación, hacia los extremos de la zona de aprovechamiento actual.

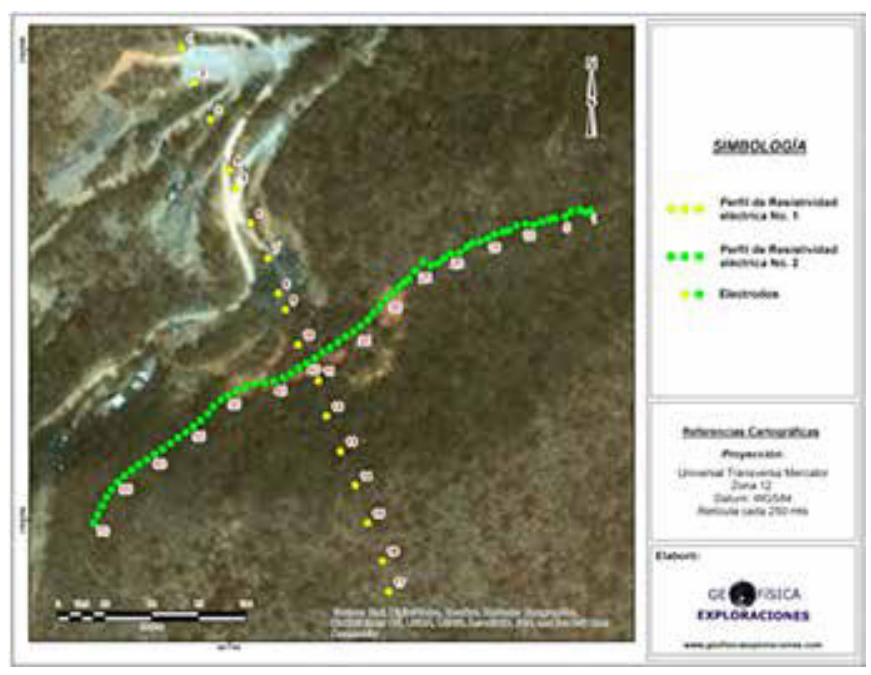

Figura 2. Plano general de localización de los datos geofísicos, los puntos verdes representan la ubicación de los sitios del perfil de Tomografía Eléctrica.

\section{METODOLOGÍA}

El método de resistividad con corriente directa es una técnica ampliamente utilizada en la prospección minera y en otras áreas de las ciencias de la tierra (Espinoza Barreras, 1983). Este método se encuentra basado en la inyección de corriente eléctrica (directa o de baja frecuencia) en el terreno y en la medición de las diferencias de potencial asociadas al paso de la corriente a través de zonas del subsuelo con distinta conductividad eléctrica. La corriente se inyecta entre dos puntos de la superficie por medio de dos electrodos y se usan otros dos electrodos para medir la diferencia de potencial en otros dos puntos de la superficie. Conociendo la corriente inyectada, la diferencia de potencial observada y las posiciones relativas de los electrodos de inyección y de medición, se puede calcular una resistividad aparente:

$$
\rho_{a}=G \frac{\Delta V}{\mathrm{I}},
$$

donde I es la corriente inyectada en Amperes, $\Delta V$ es la diferencia de potencial medida en Volts, y $G$ es un factor geométrico, en metros, que depende del arreglo de electrodos. En un medio homogéneo $\rho_{a}$ es la resistividad del medio en Ohm-m. Pero, en general, cuando el medio es heterogéneo, la resistividad aparente es una especie de promedio ponderado de la resistividad verdadera del medio,

$$
\rho_{a}(\mathbf{r})=\int_{V^{\prime}} F\left(\mathbf{r}^{\prime}, \mathbf{r}, \rho\right) \rho\left(\mathbf{r}^{\prime}\right) d^{3} r^{\prime}
$$




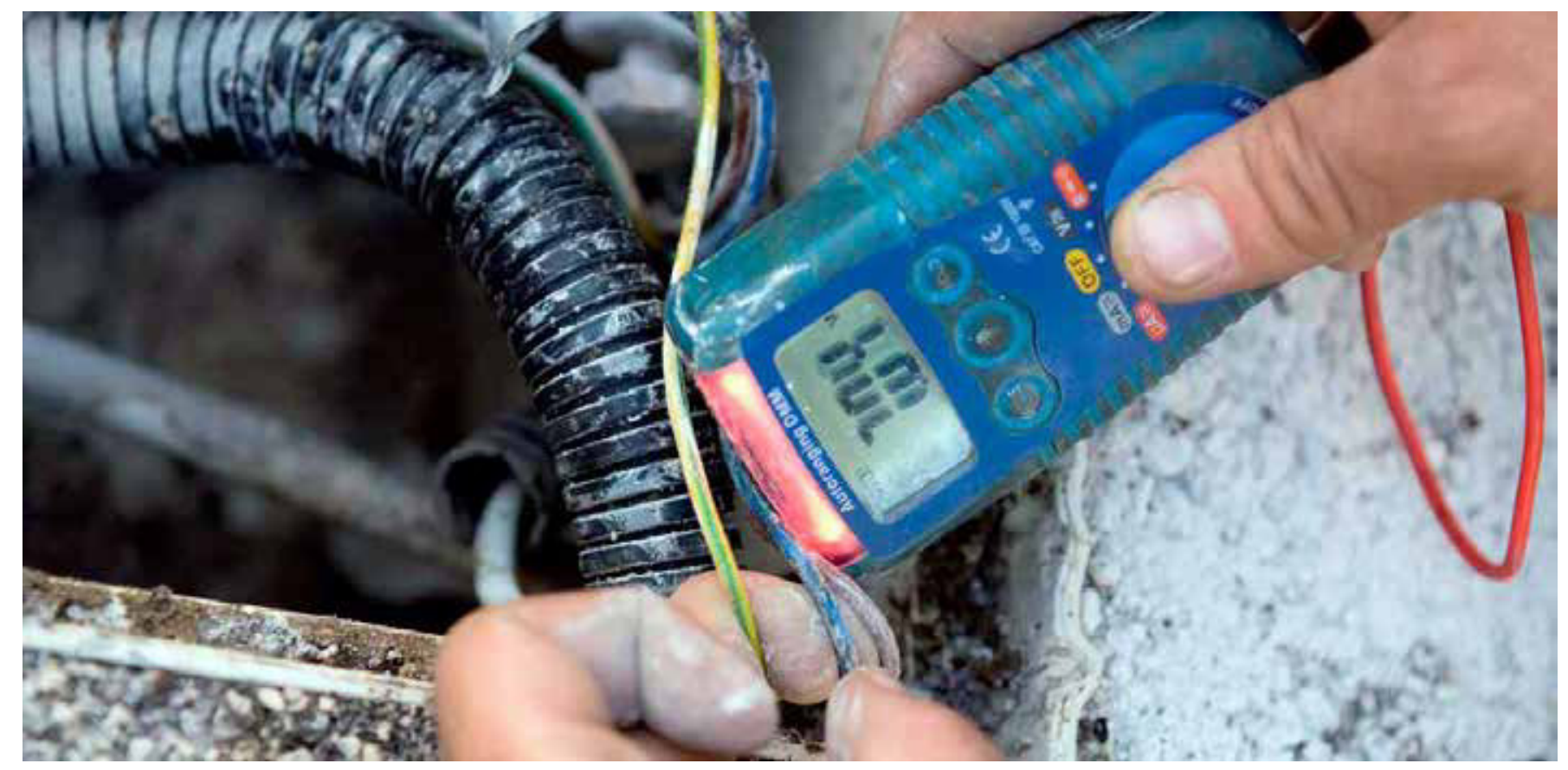

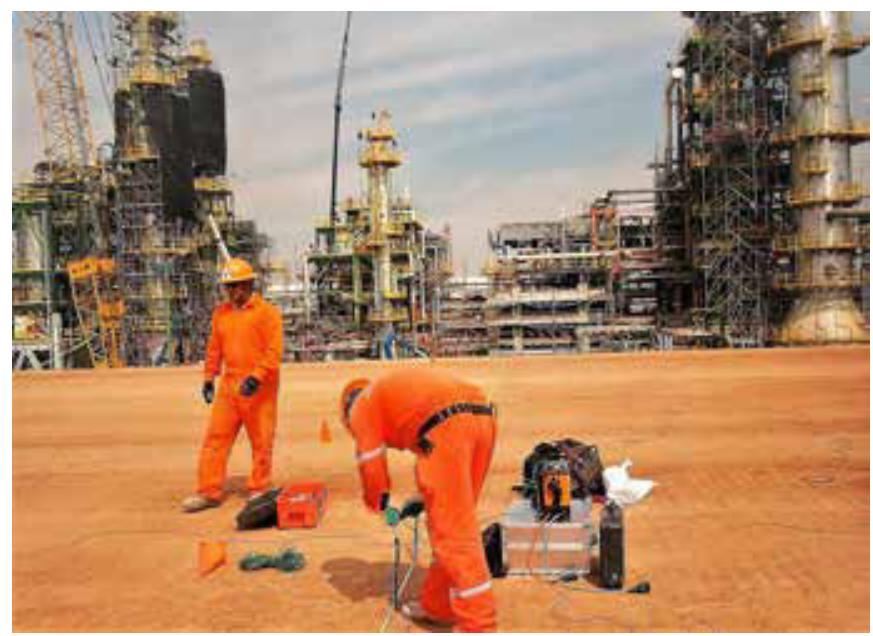

Esta relación entre la distribución espacial de la resistividad en el subsuelo $\rho\left(\mathbf{r}^{\prime}\right)$ y la resistividad aparente medida en un punto dado de la superficie es $\rho_{a}(\mathbf{r})$ generalmente no lineal, como lo muestra el hecho de que la función $F\left(\mathbf{r}^{\prime}, \mathbf{r}, \rho\right)$ dependa de $\rho$ (Gómez-Treviño, 1987).

\section{ARREGLO DIPOLO-DIPOLO}

Una de las técnicas que se usan con el método de resistividad para explorar el subsuelo a lo largo de un perfil (Figura 1), consiste en utilizar un arreglo de electrodos conocido como arreglo dipolo-dipolo. La técnica consiste en inyectar corriente mediante un par de electrodos separados entre ellos una distancia $a$, y medir la diferencia de potencial en otro par de electrodos, separados entre sí por la misma distancia $a$. El dipolo que inyecta corriente (transmisor Tx) y el que mide el potencial (receptor Rx) se encuentran separados por una distancia $n a$, donde $n$ es un número entero (Figura 2). El arreglo se mueve a lo largo del perfil conservando su geometría; para investigar a mayor profundidad, se aumenta la separación entre los dipolos Tx y Rx, incrementando el número $n$. Una vez medida la corriente inyectada y el potencial generado, se calcula la resistividad aparente usando la ecuación (1), con el factor geométrico (Orellana, 1972) G, dado por:

$$
G=\pi a n(n+1)(n+2)
$$

La resistividad aparente usualmente se grafica en forma de seudosección, en donde el eje horizontal es la distancia a lo largo del perfil y el eje vertical es una distancia proporcional a na conocida como seudo profundidad, como se muestra en el esquema de la Figura 2, en donde se puede observar que a medida que se aumenta $n$, disminuye la "cobertura" en el subsuelo. Así que en el caso extremo cuando $n=16$, se cubre un sólo punto del subsuelo.

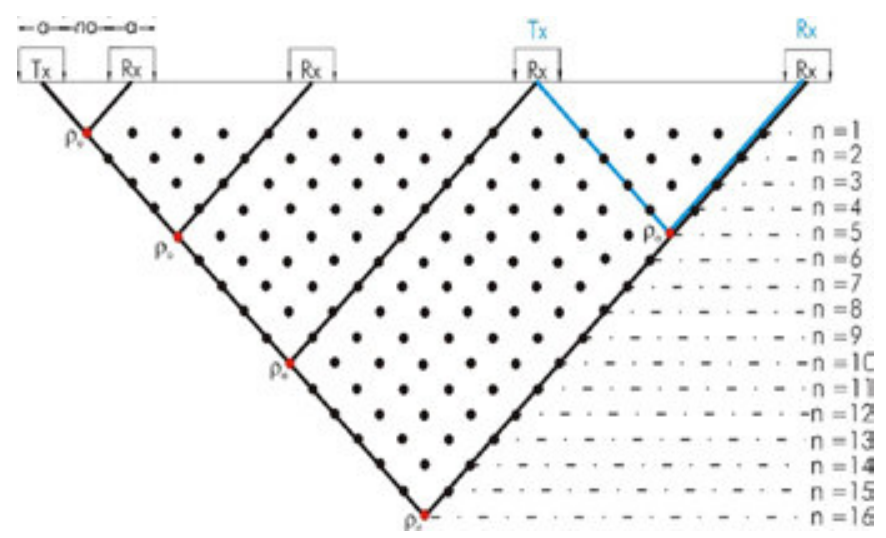

Figura 2. Esquema del funcionamiento del método de resistividad con el arreglo Dipolo-Dipolo 
La interpretación cualitativa de una seudosección de resistividad aparente (Figura 3) no es obvia, debido a la naturaleza no-lineal de la relación entre resistividad aparente $\rho_{a}(\mathbf{r})$ y resistividad del subsuelo $\rho\left(\mathbf{r}^{\prime}\right)$. Lo que puede decirse es que al aumentar $n$ (la distancia entre dipolos), aumenta la profundidad de investigación, de ahí que se denomine "seudoprofundidad" al eje vertical de la seudosección. Para $n$ pequeña la resistividad aparente está influenciada por estructuras someras, mientras que para $n$ grande, son estructuras más profundas las que afectan a la resistividad aparente.

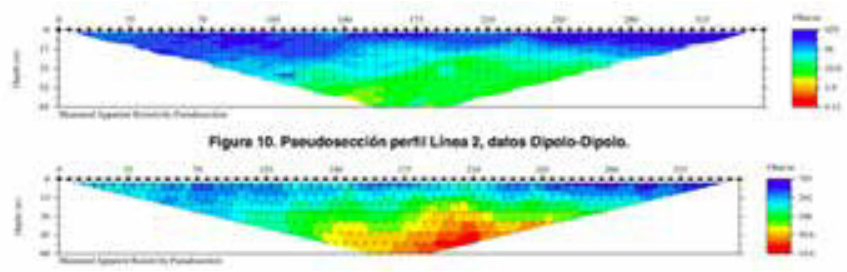

Figura 3. Seudosección de resistividad aparente obtenida con el método de dipolo-dipolo.

\section{RESULTADOS}

Las seudosecciones de isorresistividad aparente (datos crudos) se configuró utilizando el Software comercial Earthlmager 2-D (Figura 3), en la cual se puede observar cualitativamente lo siguiente:

El perfil muestra zonas o anomalías de baja resistividad (zonas en color amarillo-verde) muy focalizadas a lo largo del perfil (principalmente a profundidad) estas zonas se encuentran asociadas a la presencia de Carbón Mineral y Las anomalías de alta resistividad (zonas en color azul) se presentan principalmente en la parte superficial del perfil, la cual se encuentra relacionada con la roca encajonante.

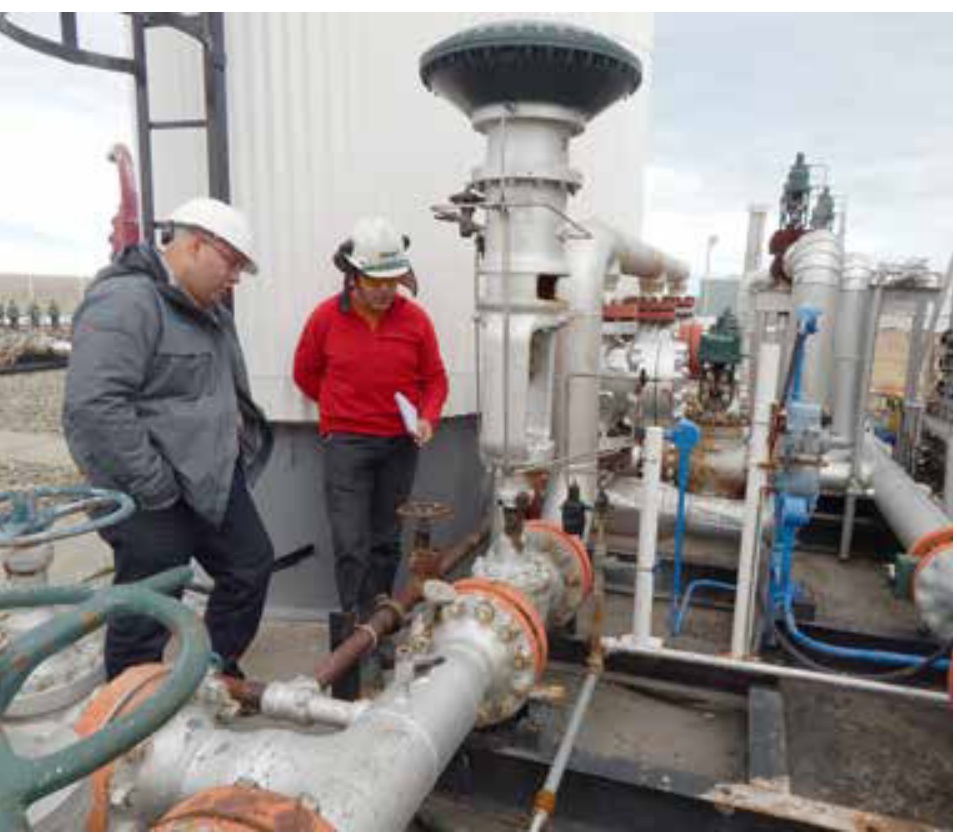

Desde el punto de vista cualitativo se puede sugerir que las zonas de baja resistividad aparente se encuentran asociadas a las zonas de Carbón mineral en explotación y sujeto a explotarse.

El equipo utilizado fue un SuperSting R8 automático de AGI Instruments con cable multi-electrodos de 70 dipolos, el cual nos proporciona información adicional al método de dipolo-dipolo, como son los sondeos eléctricos verticales (SEV's) con el arreglo Schlumberger, debido a esto, se obtuvieron tres modelos diferentes utilizando el Software comercial denominado Earthlmager 2-D de AGI Instruments asi como también el algoritmo desarrollado por Pérez-Flores et al. (2001) , primeramente se realizó un modelo para cada conjunto de datos por separado DipoloDipolo y Schlumberger (Figuras 4 y 5 ) y un tercer modelo combinando ambos conjuntos de datos (Figura 6).

La figura 4 muestra el modelo de la distribución de resistividad en el subsuelo utilizando los datos del arreglo Dipolo-Dipolo, donde se puede observar la presencia de dos zonas principales de baja resistividad, la primera entre los dipolos 28 a 36 y la segunda entre los dipolos 43 al 56, ambas asociadas a la presencia del yacimiento de Carbón Mineral; ubicadas alrededor de la cota de 753 metros sobre el nivel del mar (msnm).

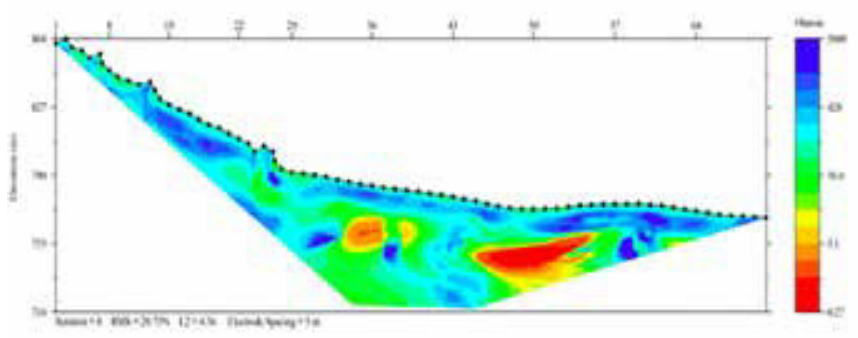

Figura 4. Modelo de resistividad utilizando datos Dipolo-Dipolo.

En el modelo de resistividad obtenido con los datos del arreglo Schlumberger (Figura 5), es posible observar la presencia de dos zonas principales de baja resistividad la primera entre los dipolos 34 a 42 y la segunda entre los dipolos 42 al 57 asociado a la presencia del yacimiento de Carbón Mineral; estas anomalías se correlacionan con las identificadas en el modelo de los datos de Dipolo-Dipolo (Figura 4); ambas zonas se ubican a la misma profundidad.

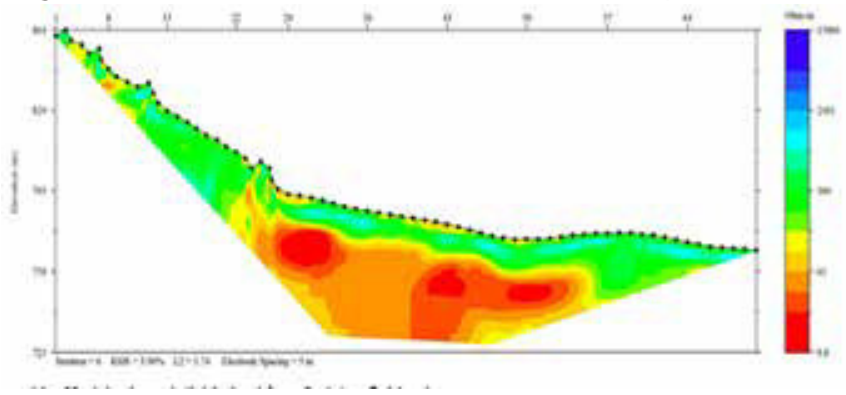

Figura 5. Modelo de resistividad utilizando sondeos eléctricos verticales con arreglo Schlumberger. 
El modelo de la figura 6 muestra la distribución de resistividad en el subsuelo utilizando los datos de ambos arreglos, en el cual se observa la presencia de dos áreas de baja resistividad identificadas en los modelos anteriores pero mayormente focalizadas; este modelo presenta a mayor detalle los cuerpos de carbón mineral en el subsuelo siendo tres las zonas principales identificadas en el modelo por los círculos de color rojo: la primer zona se encuentra localizada entre los dipolos 29-34 a una elevación de 790 msnm, la segunda zona se encuentra entre los dipolos 3542 a una elevación de 770 msnm y la tercer zona que es la de mayor tamaño se encuentra localizada entre los dipolos 43-55 a una elevación de 750 msnm.

También se identifican cuatro zonas de alta resistividad eléctrica en los alrededores de los cuerpos de Carbón, indicados en el modelo de la figura 6 con los círculos de color negro, estas zonas se encuentran posiblemente asociados a la presencia de cavidades (túneles) utilizados para la explotación del yacimiento mineral y/o cuerpos de roca encajonante, las cuales se encargan de cortar la secuencia de carbón a profundidad.

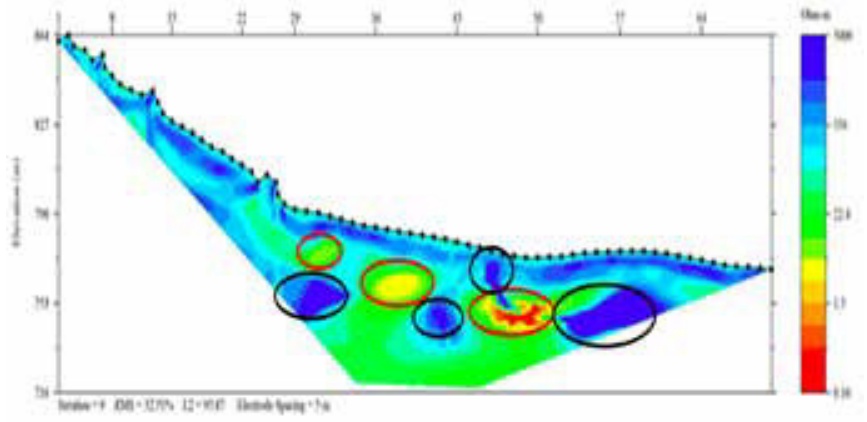

Figura 6. Modelo de resistividad, utilizando la inversión conjunta de los dos tipos de datos dipolo-dipolo y

Schlumberguer (los círculos de color rojo identifican las zonas de carbón mineral y los círculos de color negro la presencia de túneles y/o roca encajonante).

\section{CONCLUSIONES}

Con base a los resultados expuestos con anterioridad se obtienen las siguientes conclusiones y recomendaciones:

"De acuerdo a lo observado en la superficie del terreno, área de estudio se encuentra conformada litológicamente por depósitos sedimentarios (areniscas y lutitas) donde se localiza el Carbón Mineral que actualmente se explota en el predio y a rocas ígneas que sobreyacen a las sedimentarias.

-Del análisis de los modelos de resistividad obtenidos, se observaron anomalías de baja resistividad eléctrica (alta conductividad) a lo largo del perfil, asociados a la presencia de carbón Mineral actualmente en explotación.

"Estas anomalías de baja resistividad se encuentran dentro de un medio homogéneo de resistividades alrededor de 30 ohm-m lo que permite su identificación.

- Las zonas de alta resistividad encontradas en superficie están asociadas a la presencia de material aluvial y zonas de conglomerados y a profundidad con la presencia de cavidades o túneles utilizadas para explotar los cuerpos de Carbón así como a cuerpos de roca encajonante.

Se identificaron tres zonas principales asociadas a la presencia de carbón mineral

- Se observa la presencia de zonas de alta Resistividad que cortan lo cuerpos de carbón y/o se ubican en dirección a los cuerpos lo que nos hace inferir que son cavidades o túneles utilizados para la explotación del Mineral.

\section{BIBLIOGRAFIA}

[1] Espinoza Barreras, F. 1983.Estudio de resistividad en los valles San Pedro Mártir y Valle Chico, Baja California. Tesis de Maestría en Ciencias. Centro de Investigación Científica y de Educación Superior de Ensenada, Baja California. 112 hojas.

[2] Gomez Treviño, E. 1987. "Non-linear integral equations for electromagnetic inverse problems". Geophysics 52: 1297$1302 \mathrm{p}$.

[3] INEGI. Carta Topográfica escala 1:50000.Hoja Tecoripa. Clave H12D64. 2a edición. SGM. Carta Geológica escala 1:50000. Hoja Tecoripa. Clave H12D64. 2a edición.

[4] Newman G.A., Gasperikova E., Hoversten G.M., Wannamaker P.E., 2008. Three-dimensional magnetotelluric characterization of the Coso geothermal field. Geothermics 37, 369-399 p.

[5] Orellana, E. 1972. "Prospección Geoeléctrica en corriente continua". Ed. Paraninfo Madrid. 523 p.

[6] Pérez-Flores M. A. y E. Gómez Treviño, 2001, Imaging lowfrecuency and dc electromagnetic fields using a simple linear aproximation. Geophysics, 66,1067-1081.

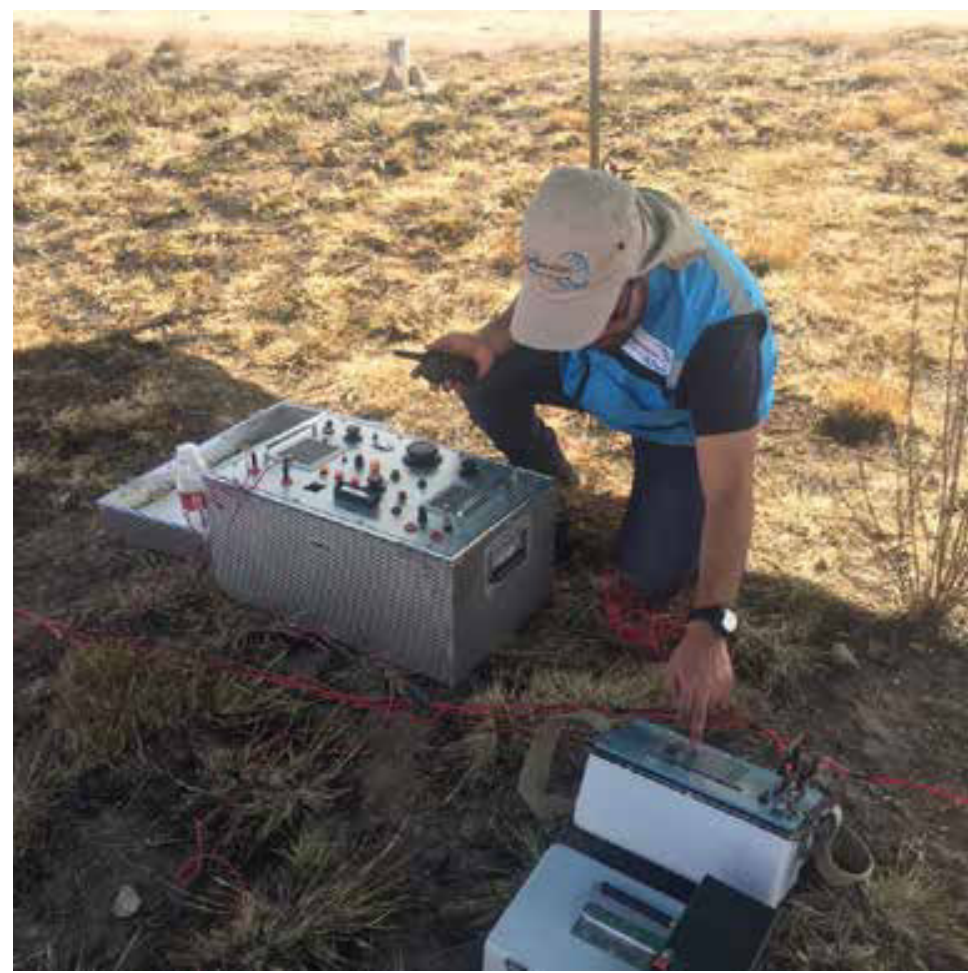

